\title{
Efficient CNFET-based Rectifiers for Nanoelectronics
}

\author{
Mohammad Hossein Moaiyeri \\ Nanotechnology and Quantum \\ Computing Lab., Shahid \\ Beheshti University, G.C., \\ Tehran, Iran
}

\author{
Keivan Navi \\ Faculty of Electrical and \\ Computing Engineering, Shahid \\ Beheshti University, G.C., \\ Tehran, Iran
}

\author{
Omid Hashemipour \\ Faculty of Electrical and \\ Computing Engineering, Shahid \\ Beheshti University, G.C., \\ Tehran, Iran
}

\begin{abstract}
By scaling down the feature size of CMOS technology deeply in nanoranges, many problems and challenges, such as short channel effects, will restrict its usefulness for the near future robust and energy efficient applications. The CNFET nanodevice seems to be a feasible alternative for bulk CMOS due to its unique electrical properties and similarities with MOSFET. In this paper, efficient CNFET-based analog inverter, half-wave rectifier and full-wave rectifier circuits are proposed for nanoelectronics. The proposed rectifiers are designed based on a CNFET-based 2-transistor analog inverter and a 2-transistor MAX structure. While the previous designs have suffered from a high number of transistors or threshold loss problem, the unique properties of CNFET nanodevice, such as tunable threshold voltage, are utilized in the proposed circuits to reach efficient, full-swing and highprecision designs. The proposed circuits are simulated using HSPICE based on the standard MOSFET-like CNFET model, valid for $\geq 10 \mathrm{~nm}$ technology nodes and the functionalities are verified with both DC and transient analyses.
\end{abstract}

\section{Keywords}

Nanoelectronics, Carbon nanotube field effect transistor (CNFET), rectifier, Analog inverter

\section{INTRODUCTION}

As the channel length scaled down deeply in nanoranges, different negative effects impressed the current-voltage characteristics of silicon MOSFET family which led to very lower output resistance and device transconductance that restricted the miniaturization of the feature size of this family. Moreover, scaling down the MOSFET technology has resulted in many problems such as reduced gate control, shortchannel effects, velocity saturation, carrier mobility degradation, exponentially rising leakage currents [1]. As a result, many emerging nanoscale devices, such as quantumdot cellular automata (QCA), single electron transistor (SET), Benzene ring FET and carbon nanotube FET (CNFET) have been presented to replace the CMOS technology [2-6]. Considering these nanodevices, CNFET is the most encouraging option for replacing the Si MOSFET in the time to come because of its inherent similarities with conventional MOSFET and the existence of both $\mathrm{N}$-type and P-type transistors, which have led to comparable designing techniques with CMOS technology [6]. In addition, comparing with MOSFET, CNFET is quite faster, consumes lower power and has same electron and hole mobilities $\left(\mu_{\mathrm{N}}=\mu_{\mathrm{P}}\right)[1,6]$.

Many applications such as Full Adder cells, MVL circuits, and fuzzy min-max circuits have been designed based on CNFETs [7-9]. However, due to CNFET unique characteristics it can be very useful for designing efficient and high-performance analog circuits.
One of the most widely used analog circuits is voltage rectifier. In general, a rectifier is an electrical circuit that converts an AC signal to a DC signal and the process is called rectification. Rectifiers are generally classified into two types: half-wave rectifier and full-wave rectifier. In half wave rectification of a single-phase supply, one of the positive half or the negative half of the AC input signal is passed and the other half is obstructed. In full wave rectification, both polarities of the input signal are converted to DC.

Classically, rectifiers have widely been used to generate a DC voltage, for use as a source of power, or radio signals detectors [10].

A classical implementation of the rectifier circuit is the wellknown four-diode bridge design, shown in Figure 1. However, it is not useful for current on-chip applications. Moreover, the output has $2 V_{D}$ (diode threshold) drop, which makes this design totally unsuitable for current low voltage applications.

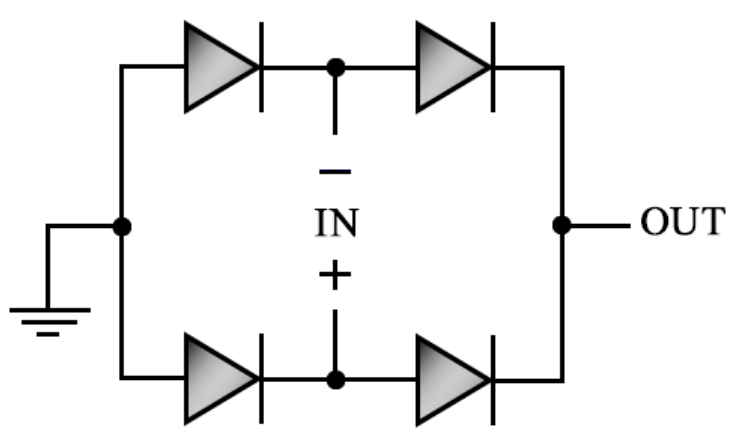

Fig 1: The well-known full-wave bridge rectifier

Using Schottky diodes can reduce the diode threshold [11] but $2 V_{D}$ drop is still intolerable. Actually, no DC voltage can be achieved if $\mathrm{V}_{\mathrm{IN}}<2 \mathrm{~V}_{\mathrm{D}}$.

An effective solution based on CMOS technology has been presented in [10] to overcome the output voltage drop. However, in this approach, due to utilizing only PMOS transistors at the output stage, the output does not include voltages smaller than $\left|\mathrm{Vth}_{\mathrm{P}}\right|$, which results in a non-full swing output and consequently underestimating the average output voltage.

In this work, efficient full-swing rectifier and analog inverter circuits are proposed for low-voltage on-chip applications based on the unique properties of CNFET nano devices.

The rest of the paper is organized as follows: In section 2, a brief review of carbon nanotube FET is provided. Section 3 describes the proposed analog inverter and rectifier circuits and includes the simulation results and finally, section 4 concludes the paper. 


\section{A REVIEW OF CARBON NANOTUBE FET (CNFET) AND ITS SPICE MODEL}

Carbon Nanotube (CNT) is a carbon allotrope which can be considered as a graphene sheet rolled into a cylindrical nanostructure. CNTs can be categorized into single-walled CNTs (SWCNTs), fabricated of a single tube, and multiwalled CNTs (MWCNTs), fabricated of more than one nested tubes [12]. A vector, called chirality vector, is defined for a CNT, which determines the formation angle of the carbon atoms along the nanotube. The chirality vector is defined by $\left(n_{1}, n_{2}\right)$ pair called chiral number. Many of the physical and electrical properties of a CNT depend on its chirality vector. A SWCNT is metallic if $n_{1}-n_{2}=3 k \quad(k \in \square)$ and otherwise it is semiconducting [12]. The semiconducting SWCNTs can be used as the channel of a CNFET device.

The structure of a typical MOSFET-like CNFET [13-15] is demonstrated in Figure 2.

According to Figure 2(a), the gate width of a CNFET can be calculated based on Equation 1 [13], in which $W_{\min }$ is the minimum gate width, determined by lithography, $N$ is the number of nanotubes underneath the gate and Pitch is the distance between the centers of two neighbor CNTs below the same gate.

$W_{\text {gate }} \approx \operatorname{Max}\left(W_{\text {min }}, N \times\right.$ Pitch $)$

Similar to a MOSFET, a CNFET has also a threshold voltage $\left(V_{\mathrm{th}}\right)$, which is required for turning on the transistor electrostatically through the gate. One of the most important and practical characteristics of a CNFET device, which makes it very suitable for designing efficient and high-performance digital and analog circuits, is the capability of determining its threshold voltage by adopting a relevant diameter for its nanotubes. The diameter of the channel CNTs $\left(D_{\mathrm{CNT}}\right)$ directly affects the bandgap and consequently the threshold voltage of the CNFET. The threshold voltage of a CNFET is approximately considered to the first order as the half bandgap of its CNTs and is calculated based on the following equation [13]:

$V_{\mathrm{th}} \approx \frac{E_{\mathrm{bg}}}{2 e}=\frac{a_{0} V_{\pi}}{e D_{\mathrm{CNT}}} \approx \frac{0.43}{D_{\mathrm{CNT}}(\mathrm{nm})}$

Where, $e$ is the unit electron charge, $E_{\mathrm{bg}}$ is the CNT bandgap, $a_{0}(\approx 0.142 \mathrm{~nm})$ is the carbon to carbon bond length in a CNT and $V_{\pi}(\approx 3.033 \mathrm{eV})$ is the carbon $\pi-\pi$ bond energy in the tight bonding model.

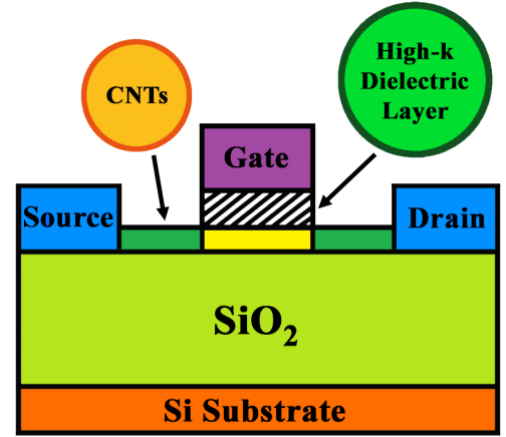

(a)

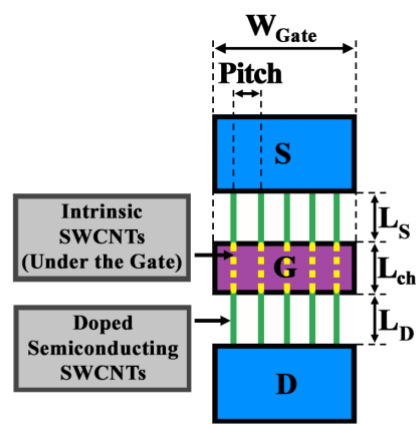

(b)

Fig 2: Structure of a MOSFET-like CNFET device (a) Cross section view (b) Top view

It can be concluded from Equation 2 that the threshold voltage of a carbon nanotube transistor is inversely proportional to the diameter of its CNTs, which is calculated based on the CNT chiral number $(n, m)$ according to the following equation [13]:

$D_{\mathrm{CNT}}=\frac{\sqrt{3} a_{0} \sqrt{n_{1}^{2}+n_{1} n_{2}+n_{2}^{2}}}{\pi} \approx 0.0783 \sqrt{n_{1}^{2}+n_{1} n_{2}+n_{2}^{2}}$

For instance, $D_{\mathrm{CNT}}$ of a CNFET with the chiral number $(n, m)$ $=(29,0)$ is $2.27 \mathrm{~nm}$ and consequently its threshold voltage is $0.192 \mathrm{~V}$.

In this work, we benefit from the unique properties of multidiameter MOSFET-like CNFETs for designing an efficient voltage rectifier for on-chip applications. MOSFETlike CNFET nanodevice has been modelled for SPICE in [1315]. This standard model has been designed for enhancementmode unipolar MOSFET-like CNFET which can include one or more semiconducting CNTs as its channel. This model considers a circuit-compatible and realistic CNFET structure and includes device nonidealities, parasitics, inter-CNT charge screening effects, Schottky-barrier effects at the contacts, scattering, back-gate effect, Source/Drain, Gate resistances and capacitances and doped source/drain expansion regions. Furthermore, the model includes a full transcapacitance network for more exact transient and dynamic performance simulations. The parameters of this CNFET model, used in this work, and their values, with brief descriptions, are listed in Table 2. The proposed designs in this work are simulated based on this model using Synopsis HSPICE with $-0.45 \mathrm{~V}$ and $0.45 \mathrm{~V}$ power supplies at room temperature. 
Table 1 Characteristics of the used CNFET SPICE model

\begin{tabular}{|c|c|c|}
\hline Parameter & Brief description & Value \\
\hline$L_{\mathrm{ch}}$ & Physical channel length & $\geq 10 \mathrm{~nm}$ \\
\hline$L_{\mathrm{sS}}$ & $\begin{array}{l}\text { The length of doped CNT source- } \\
\text { side extension region }\end{array}$ & $\geq 10 \mathrm{~nm}$ \\
\hline$L_{\mathrm{dd}}$ & $\begin{array}{l}\text { The length of doped CNT drain- } \\
\text { side extension region }\end{array}$ & $\geq 10 \mathrm{~nm}$ \\
\hline$L_{\text {geff }}$ & $\begin{array}{l}\text { The Scattering mean free path in } \\
\text { the intrinsic CNT channel and S/D } \\
\text { regions }\end{array}$ & $100 \mathrm{~nm}$ \\
\hline Pitch & $\begin{array}{l}\text { The distance between the centers } \\
\text { of two neighboring CNTs within } \\
\text { the same device }\end{array}$ & $\geq 4 \mathrm{~nm}$ \\
\hline$L_{\text {eff }}$ & $\begin{array}{l}\text { The mean free path in } \mathrm{p}+/ \mathrm{n}+ \\
\text { doped CNT. }\end{array}$ & $15 \mathrm{~nm}$ \\
\hline sub_pitch & Sub-lithographic pitch & $4 \mathrm{~nm}$ \\
\hline$K_{\mathrm{ox}}$ & $\begin{array}{l}\text { The dielectric constant of high-k } \\
\text { top gate dielectric material }\left(\mathrm{HfO}_{2}\right)\end{array}$ & 16 \\
\hline$T_{\mathrm{ox}}$ & $\begin{array}{l}\text { The thickness of high-k top gate } \\
\text { dielectric material }\end{array}$ & $4 \mathrm{~nm}$ \\
\hline$K_{\text {sub }}$ & $\begin{array}{c}\text { The dielectric constant of } \\
\text { substrate }\left(\mathrm{SiO}_{2}\right)\end{array}$ & 4 \\
\hline$C_{\text {sub }}$ & $\begin{array}{l}\text { The coupling capacitance between } \\
\text { the channel region and the } \\
\text { substrate }\left(\mathrm{SiO}_{2}\right)\end{array}$ & $17 \mathrm{aF} / \mu \mathrm{m}$ \\
\hline Efi & $\begin{array}{l}\text { The Fermi level of the doped S/D } \\
\text { tube }\end{array}$ & $6 \mathrm{eV}$ \\
\hline phi_M & $\begin{array}{c}\text { The work function of } \\
\text { Source/Drain metal contact }\end{array}$ & $4.6 \mathrm{eV}$ \\
\hline phi_S & CNT work function & $4.5 \mathrm{eV}$ \\
\hline
\end{tabular}

\section{THE PROPOSED DESIGNS}

One interesting approach for designing half-wave and fullwave rectifiers is based on analog maximum circuit [16]. As shown in Figure 3(a), for a half wave rectification, a sinusoidal wave $\left(V_{1}=V_{i} \operatorname{Sin} \omega t\right)$ is fed into the first input of the maximum circuit and the second input is connected to the ground. As a result only the positive cycles of V1 are transferred to output and half-wave rectification $\left(V_{O}=\left.V_{1}\right|_{V_{1} \geq 0}\right)$ is obtained.

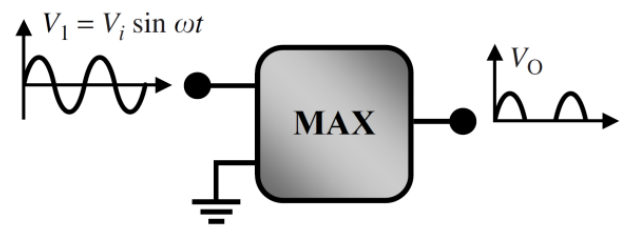

(a)

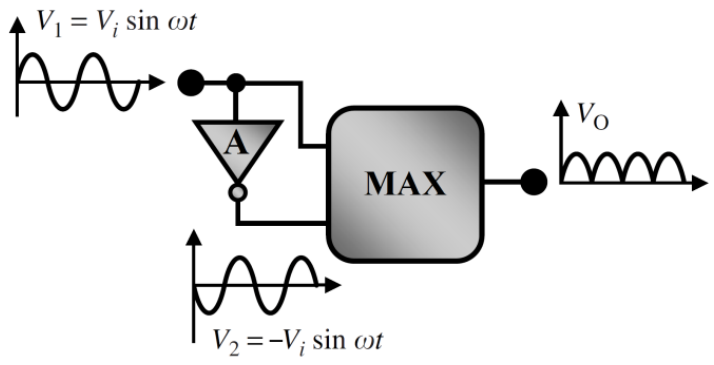

(b)

Fig 3: Designing rectifier circuits based on analog MAX circuit (a) Half-wave rectifier (b) Full-wave rectifier
Furthermore, as demonstrated in Figure 3(b), for a full wave rectification, a sinusoidal signal with $180^{\circ}$ phase difference with the first input signal $\left(V_{2}=-V_{i} \operatorname{Sin} \omega t\right)$ is fed to the second input. The required $180^{\circ}$ phase difference can be provided by means of an analog inverter. Accordingly, the half negative and positive cycles of both inputs are overlapped and only positive cycles of $V_{1}$ and $V_{2}$ are transferred to the output and full-wave rectification $\left(V_{O}=\left.V_{1}\right|_{V_{1} \geq 0}+\left.V_{2}\right|_{V_{2} \geq 0}\right)$ is obtained.

Two key points in implementing this method at transistor level are efficiency of the maximum circuit and rail-to-rail operation of the analog inverter. As the inputs of the MAX circuit for half-wave and full-wave rectifications are specific inputs, a very simpler circuit can be used instead of a complex MAX circuit.

The proposed $16 \mathrm{~nm}$ CNFET-based half-wave rectifier, composed of only two P-CNFETs, is shown in Figure 4. As in a single P-FET $\mathrm{V}_{\text {OUT }}=\operatorname{Max}\left(\mathrm{V}_{\mathrm{IN}},\left(\mathrm{V}_{\mathrm{G}}-\mathrm{V}_{\mathrm{thP}}\right)\right)$, the positive cycles of the input signal are passed to the output through $\mathrm{P}_{1}\left(\mathrm{P}_{2}\right.$ is $\mathrm{OFF}$ ) and when the input is negative the output remains zero through $\mathrm{P}_{2}\left(\mathrm{P}_{1}\right.$ is $\left.\mathrm{OFF}\right)$. According to Equation 2, using CNFETs with large diameters eliminates the voltage drop, leads to a full-swing output and enhances the speed and the driving capability of the circuit. Moreover, by adopting the suitable number of tubes under the gate $(N)$, the desired driving power can be met. In this design, $L_{\mathrm{ch}}=L_{\mathrm{S}}=L_{\mathrm{D}}=16 \mathrm{~nm}$, Pitch $=20 \mathrm{~nm}, D_{\mathrm{CNT}}=3.132 \mathrm{~nm}$ and $N=12$.

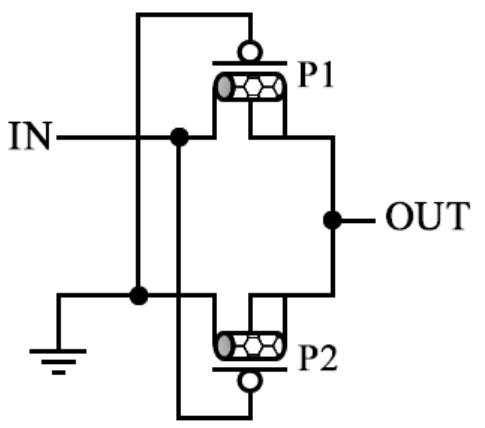

Fig 4: The proposed CNFET-based half-wave rectifier

The proposed efficient CNFET-based full-wave rectifier, composed of only 4 transistors, is shown in Figure 5 . Although a single P-FET has the definition of the maximum function inherently $\left(\mathrm{V}_{\mathrm{OUT}}=\operatorname{Max}\left(\mathrm{V}_{\mathrm{IN}},\left(\mathrm{V}_{\mathrm{g}}-\mathrm{V}_{\text {thP }}\right)\right)\right)$, the output voltage is correct only when the gate voltage is less than or equal to the drain voltage. As a result, two P-CNFETs should be used such as shown in Figure 2. Moreover, as a general MAX circuit, in some cases, such as when both inputs are high, the output will be floating. However, this situation does not occur at all due to $180^{\circ}$ phase difference between the inputs of the full-wave rectifier. In this situation, at least one of the transistors is $\mathrm{ON}$ and passes the correct voltage to the output node. According to Equation 2, using CNFETs with large diameters leads to elimination of the voltage drop problem and improvement of both driving capability and speed of the circuit. In this design for $\mathrm{P}_{1}$ and $\mathrm{P}_{2}$ CNFETs, $L_{\mathrm{ch}}=L_{\mathrm{S}}=L_{\mathrm{D}}=16 \mathrm{~nm}$, Pitch $=20 \mathrm{~nm}, D_{\mathrm{CNT}}=3.132 \mathrm{~nm}$ and $N=12$.

In order to provide an $180^{\circ}$ phase difference, an analog inverter is proposed which is designed based on the unique properties of the CNFET nanodevice. The proposed analog inverter is composed of two CNFETs indicated with $\mathrm{T}_{1}$ and $\mathrm{T}_{2}$ in Figure 5. 


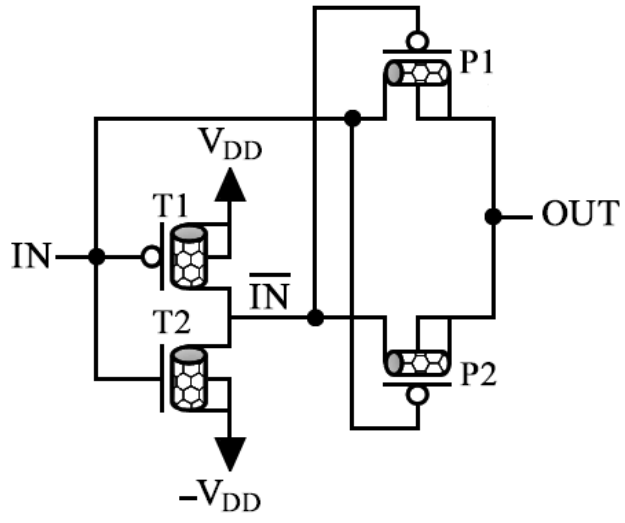

Fig 5: The proposed CNFET-based full-wave rectifier

The main idea is to modify the behavior of a digital inverter to obtain an analog inverter, by keeping the transistors in saturation condition. This can be achieved by using CNFETs, which have adoptable threshold voltages due to the adjustability of the diameters of their CNTs. The diameter of both N-CNFET and P-CNFET transistors are set to be $4.3 \mathrm{~nm}$. Therefore, as shown in Figure 6(a), the Voltage Transfer Characteristic (VTC) curve of the analog inverter is full-swing and quite near ideal with more than 98\% accuracy. Figure 6(b) shows the transient response of the proposed analog inverter. In this design for $T_{1}$ and $T_{2}$ CNFETs, $L_{\mathrm{ch}}=L_{\mathrm{S}}=L_{\mathrm{D}}=90 \mathrm{~nm}$, Pitch $=5 \mathrm{~nm}, D_{\mathrm{CNT}}=4.3 \mathrm{~nm}$ and $N=15$.

The transient responses of the proposed designs are shown in Figure 7. According to the simulation results the proposed designs operate correctly, with full voltage swing and with high precision. In addition, the delay, average power consumption and energy consumption of the proposed fullwave rectifier are calculated as $4.6 \mathrm{ps}, 50 \mu \mathrm{W}$ and $230 \mathrm{aJ}$.

\section{Conclusion}

This paper presents efficient CNFET-based half-wave and full-wave rectifiers for on-chip nanoelectronics applications. The proposed rectifiers are designed based on analog maximum and inverter circuits. However, for implementing the MAX circuit an efficient 2-transisitor structure is utilized in the proposed designs. The previous designs have high number of transistors or suffered from $\mathrm{V}_{\text {th }}$ loss problem. However, the unique attributes of CNFET nanodevice, such as adjustable $\mathrm{V}_{\mathrm{th}}$, are utilized in the proposed circuits to obtain full-swing, efficient and high-precision designs. The proposed circuits are simulated using HSPICE based on the standard MOSFET-like CNFET model, valid for $\geq 10 \mathrm{~nm}$ nodes and their operations are verified with both DC and transient analyses.

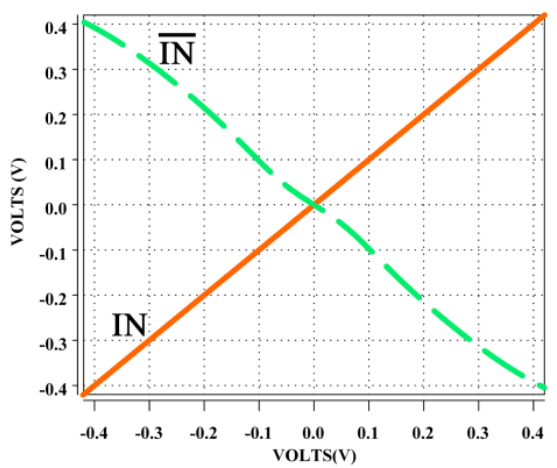

(a)

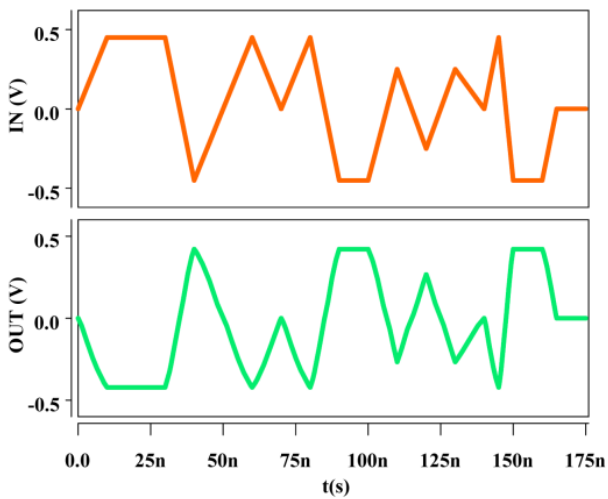

(b)

Fig 6: Functionality of the proposed analog inverter (a) DC response (b) Transient response
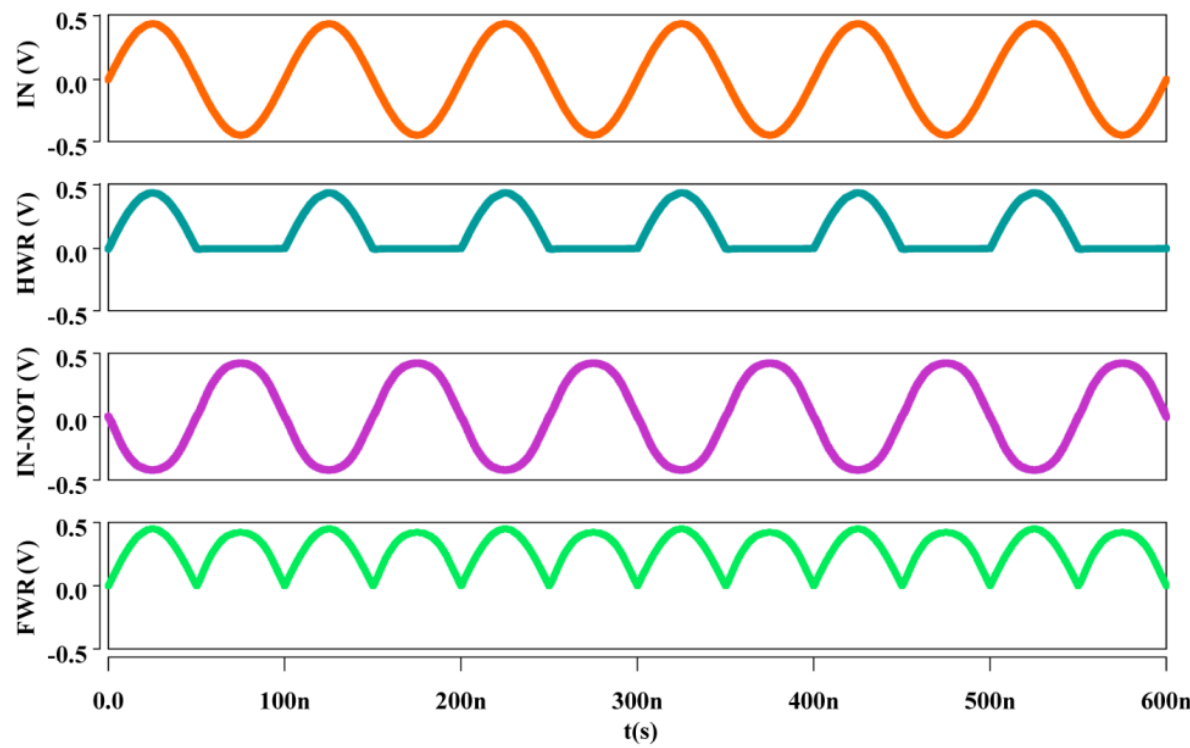

Fig 7: Transient responses of the proposed designs 


\section{ACKNOWLEDGMENTS}

This work has been supported by the Grant number 600/629 from the vice presidency of research and technology of Shahid Beheshti University, G. C.

\section{REFERENCES}

[1] Kim Y.-B., "Challenges for Nanoscale MOSFETs and Emerging Nanoelectronics," Transactions on Electrical and Electronic Materials, Vol. 11, No. 3, pp. 93-105, 2010.

[2] Sayedsalehi S., Moaiyeri M. H., Navi K., "Novel Efficient Adder Circuits for Quantum-Dot Cellular Automata," Journal of Computational and Theoretical Nanoscience, Vol. 8, No. 9, pp. 1769-1775, 2011.

[3] Navi K., Tehrani M. A., Khatami M., "Well-Polarized Quantum-dot Cellular Automata Inverters," International Journal of Computer Applications, Vol. 58, No. 20, pp. $10-13,2012$

[4] Sahafi A., Moaiyeri M. H., Navi K., Hashemipour O., "Efficient Single-Electron Transistor Inverter-Based Logic Circuits and Memory Elements," Journal of Computational and Theoretical Nanoscience, Vol. 10, 2013.

[5] Ghasemi M., Sam M., Moaiyeri M. H., Khosravi F., Navi K., "A new SPICE model for organic molecular transistors and a novel hybrid architecture," IEICE Electronics Express, Vol. 9, No. 10, pp. 926-931, 2012.

[6] Moaiyeri M. H., Doostaregan A., Navi K., "Design of Energy-Efficient and Robust Ternary Circuits for Nanotechnology," IET Circuits, Devices \& Systems, Vol. 5, No. 4, pp. 285-296, 2011.

[7] Lin S., Kim Y-B., Lombardi F., "CNTFET-Based Design of Ternary Logic Gates and Arithmetic Circuits," IEEE Transactions on Nanotechnology, Vol. 10, No. 2, pp. 217-225, 2011.

[8] Moaiyeri M. H., Faghih Mirzaee R., Navi K., Momeni A., "Design and analysis of a high-performance CNFET- based Full Adder," Taylor and Francis, International Journal of Electronics, Vol. 99, No. 1, pp. 113-130, 2012.

[9] Moaiyeri M. H., Chavoshisani R., Jalali A., Navi K., Hashemipour O., "High-performance Mixed-Mode Universal Min-Max Circuits for Nanotechnology, Circuits, Systems, and Signal Processing, Vol. 31, No. 2, pp. 465-488, 2012.

[10] Facen A., Boni A., "Power Supply Generation in CMOS passive UHF RFID tags," IEEE research in microelectronics and electronics, pp. 33-36, 2006.

[11] Karthaus U., Fischer M., "Fully integrated passive UHF RFID transponder IC with 16.7-uW minimum RF input power," IEEE Journal of Solid-State Circuits, Vol. 38, No. 10, pp. 1602-1608, 2003.

[12] McEuen P. L., Fuhrer M., Park H., "Single-Walled Carbon Nanotube Electronics," IEEE Transactions on Nanotechnology, Vol. 1, No. 1, pp. 78-85, 2002.

[13] Deng J., "Device modeling and circuit performance evaluation for nanoscale devices: silicon technology beyond $45 \mathrm{~nm}$ node and carbon nanotube field effect transistors," Doctoral Thesis, Stanford University, 2007.

[14] Deng J., Wong H.-S.P., "A Compact SPICE Model for Carbon-Nanotube Field-Effect Transistors Including Nonidealities and Its Application-Part I: Model of the Intrinsic Channel Region," IEEE Transactions on Electron Device, Vol. 54, No. 12, pp. 3186-3194, 2007.

[15] Deng J., Wong H.-S.P., "A Compact SPICE Model for Carbon-Nanotube Field-Effect Transistors Including Nonidealities and Its Application-Part II: Full Device Model and Circuit Performance Benchmarking," IEEE Transactions on Electron Device, Vol. 54, No. 12, pp. 3195-3205, 2007.

[16] Prommee P., Chattrakun K., "CMOS WTA maximum and minimum circuits with their applications to analog switch and rectifiers," Microelectronics Journal, Vol. 42, No. 1, pp. 52-62, 2011. 